\title{
Analysis of Electroencephalogram of Autism Spectrum Disorder Using Correlation Dimension Changes in brain Map
}

\section{Nahid Ghoreishi, Samane Zare Molkabad, Somayeh Baratzade, Ateke Goshvarpour, Ghasem Sadeghi Bajestani*}

\section{ABSTRACT}

Introduction: Autism spectrum disorder (ASD) is a neurodevelopmental disorder that affects information processing in the nervous system and the procedure of natural brain evolution. the processing and analysis of the brain function of these patients have captivated,Therefore the attention of many researchers. Qualitative electroencephalography (EEG) is an evaluation method for determining functional brain abnormalities that is different from quantitative EEG. Chaotic tools are used in qualitative EEG whereas linear and nonlinear methods are applied in quantitative EEG. The purpose of the present study is to compare qualitative EEG findings of healthy and autistic subjects. Materials and Methods: In this study, 19 channels Fp2, Fp1, F7, T3, T5, O1, O2, ,of brain signals (Cz, C4, F4, Fz, F3, C3, P3, Pz, P4, T4, F8 At first 5-minutes .T6) of 6 healthy and 5 autistic subjects were evaluated in two phases with closed eyes and then 5-minutes with opened eyes, the subject's EEG was recorded. After removing the artifacts, the correlation dimension of the signals was calculated, and .brain maps were plotted to analyze the changes of correlation dimension on the scalp surface Results: By comparison of the brain maps of the healthy and autistic groups between the opened and closed eyes periods, we found there was a difference between the brain function especially in the T3 and T4 regions of the temporal regions as well as frontal ,of the groups Conclusion: Using brain maps, correlation dimension mapping on and posterior areas the brain surface provides a better understanding of brain dynamics in autistic subjects.

\section{Keywords:}

1. Brain Mapping

2. Electroencephalography 3. Autism Spectrum Disorder

*Corresponding Author: Ghasem Sadeghi Bajestani

Email:g.sadeghi@imamreza.ac.ir 
تجزيه و تحليل سيخَنال الكتريكى مغزى اختلال طيف اوتيسم با استفاده از تغييرات بعدهمبستگى در نقشأهُ مغزى

ناهيد قريشى، سمانه زارع ملك آباد، سميه برات زاده، عاتكه كَشواريور، قاسم صادقى بجستانى"

كروه مهندسى يزشكى، دانشخاه امام رضا (ع)، مشهد، ايران

اطلاعات مقاله:

يذيرش: سا دى وqس|

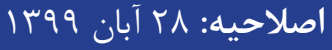

دريافت: أ تير وج

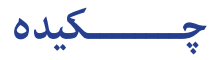

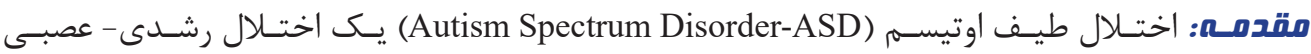

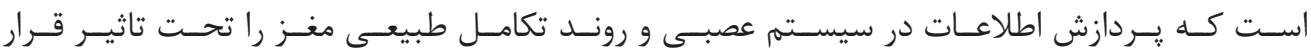

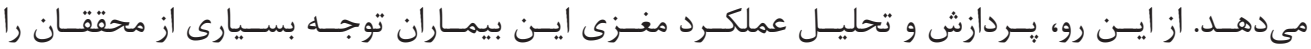

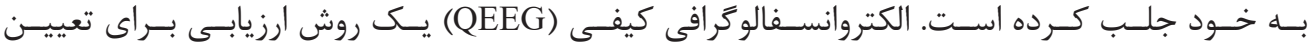

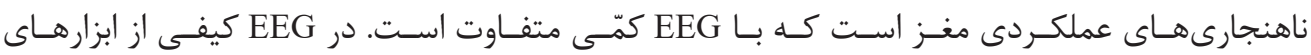

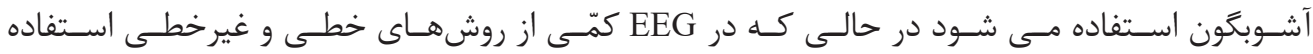

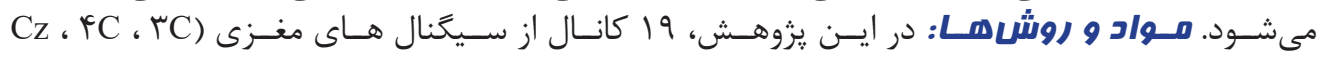

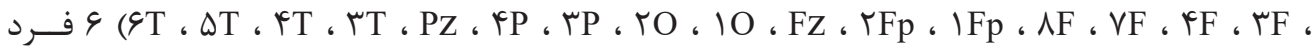

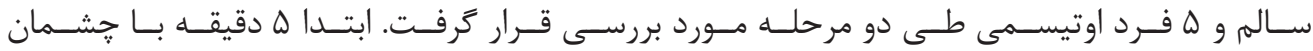

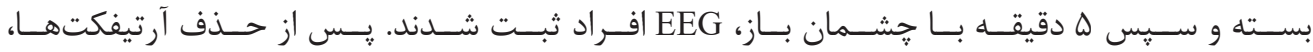

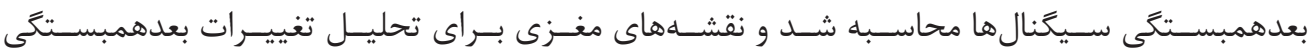

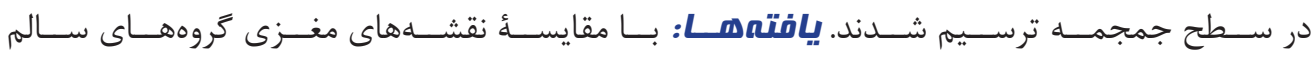

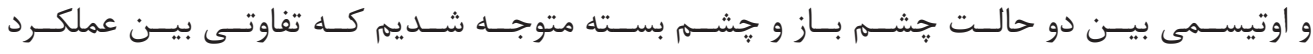

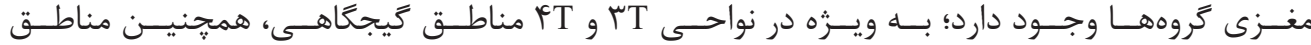

وازههاى كليدى:

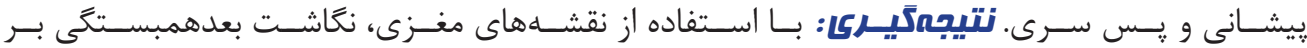

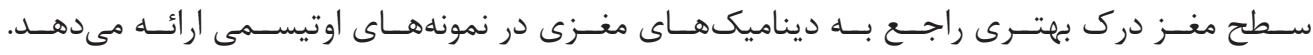

- 1 r- الكتروانسفالوخرافى ب- اختلال طيف اوتيسم
*ويسنده مسئول: قاسم صادقى بجستانى ويست الكترونيك: g.sadeghi@imamreza.ac.ir 


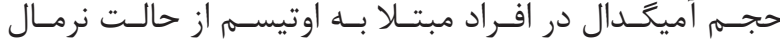

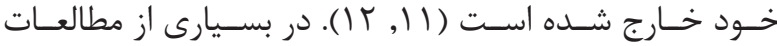

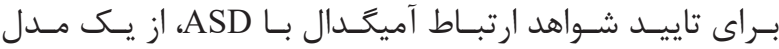

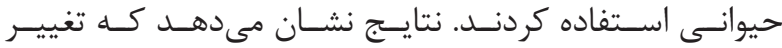

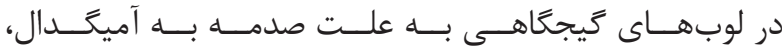

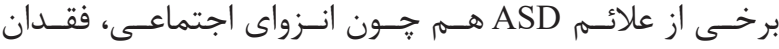

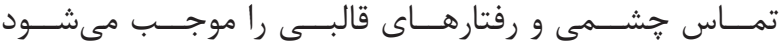

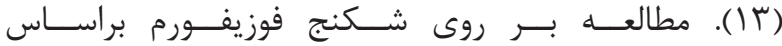

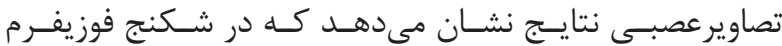

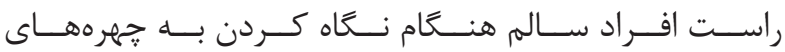

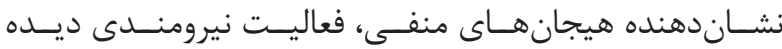

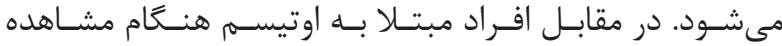

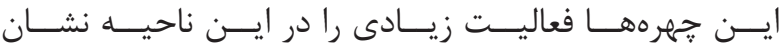

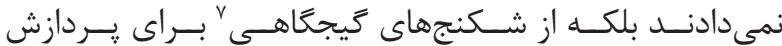

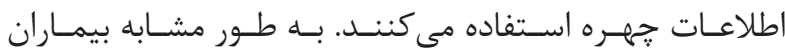

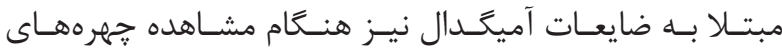

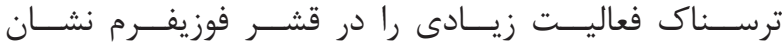

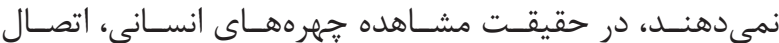

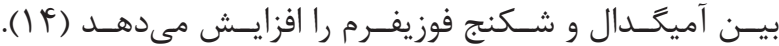

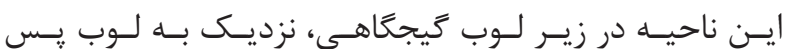

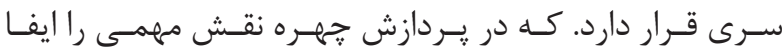

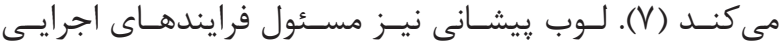

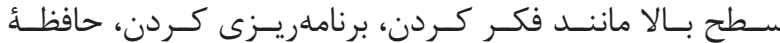

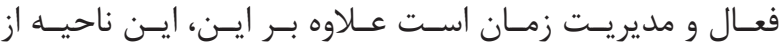

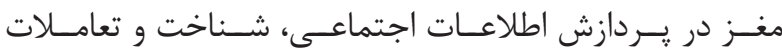

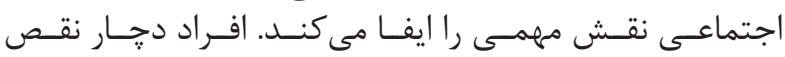

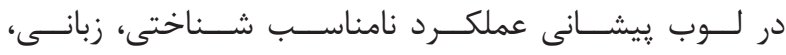

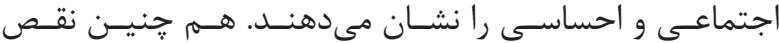

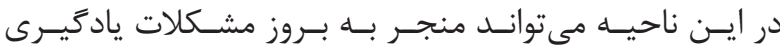

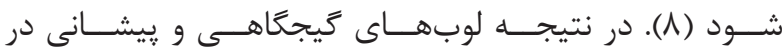

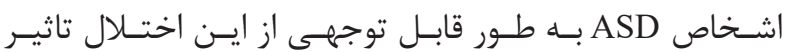

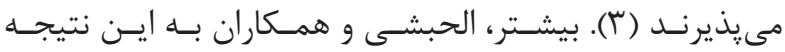

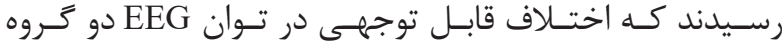

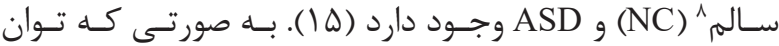

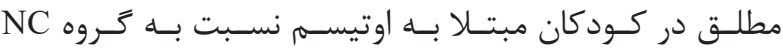

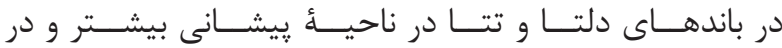

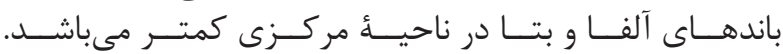

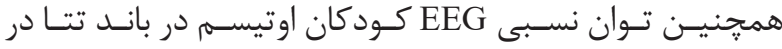

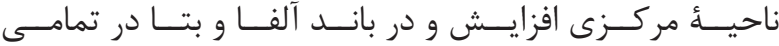

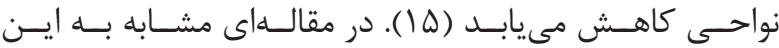

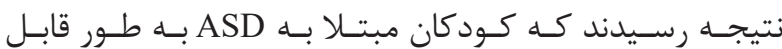

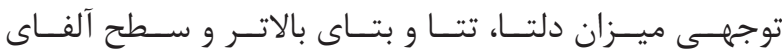

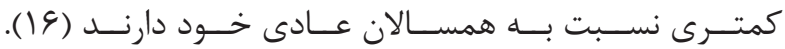

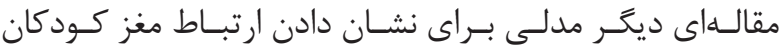

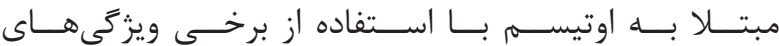

${ }^{1}$ Autism Spectrum Disorder

${ }^{2}$ Amygdala

${ }^{3}$ Fusiform sulcus

${ }^{4}$ Prefrontal cortex

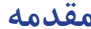

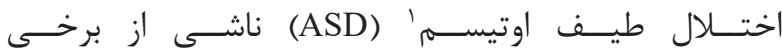

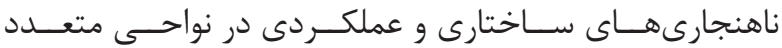

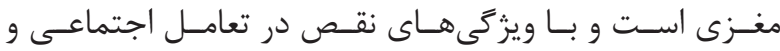

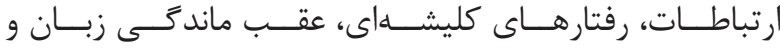

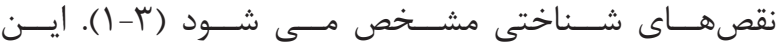

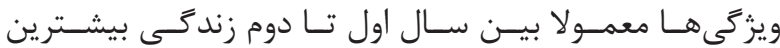

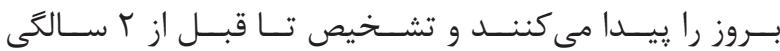

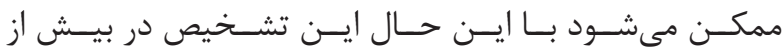

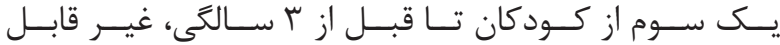

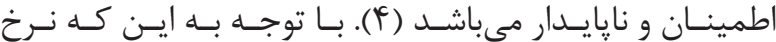

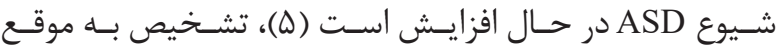

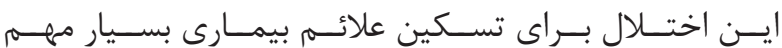

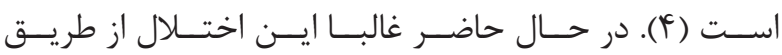

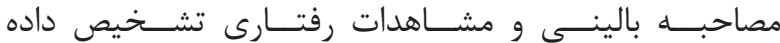

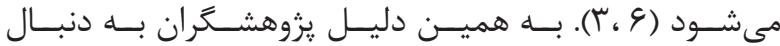

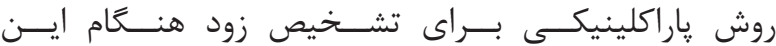

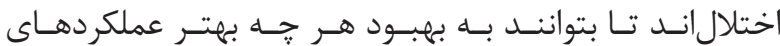

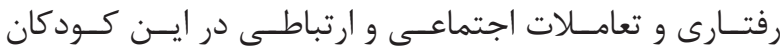

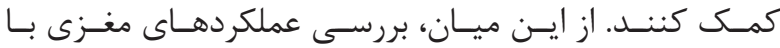

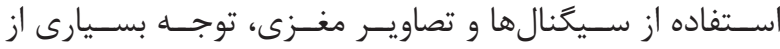

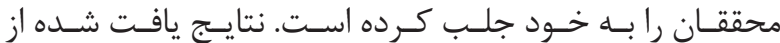

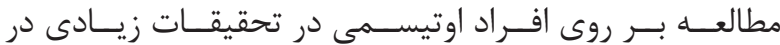

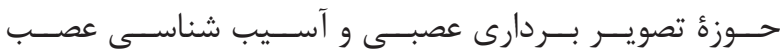

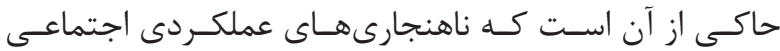

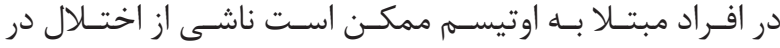

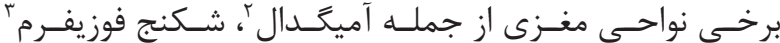

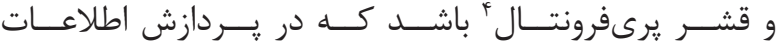

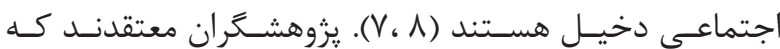

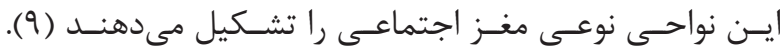

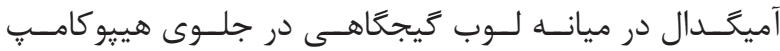

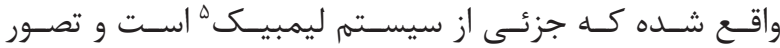

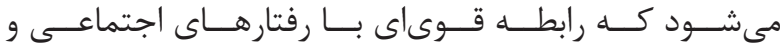

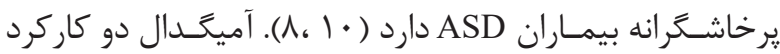

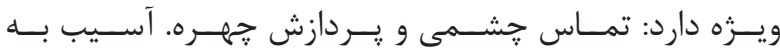

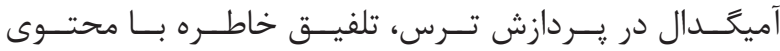

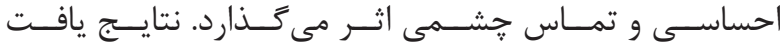

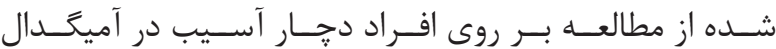

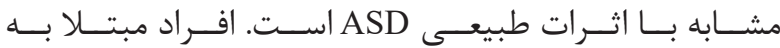

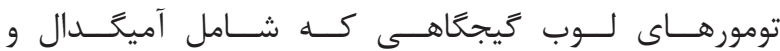

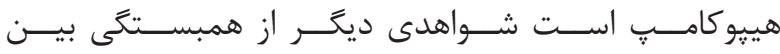

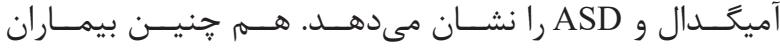

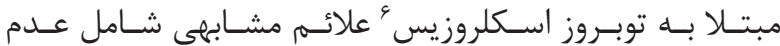

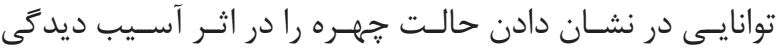

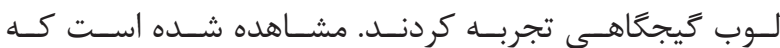

${ }^{5}$ Limbic System

${ }^{6}$ Tuberous-sclerosis

${ }^{7}$ Temporal gyrus

${ }^{8}$ Normally Control 


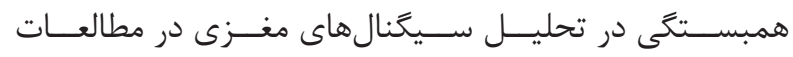

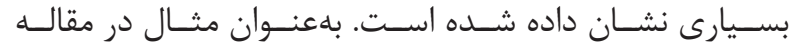

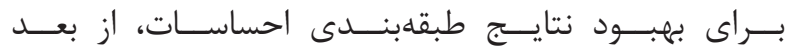

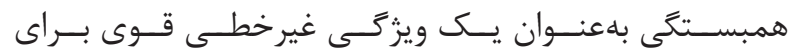

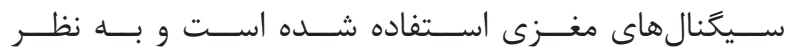

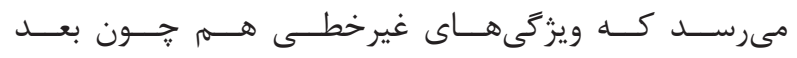

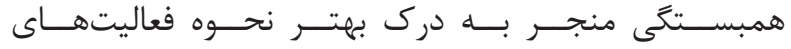

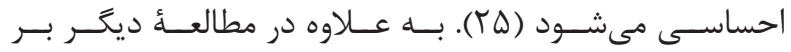

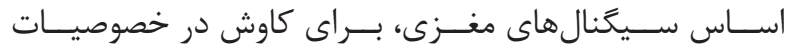

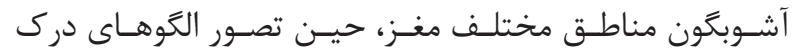

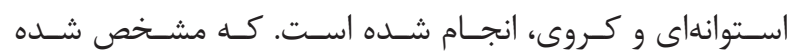

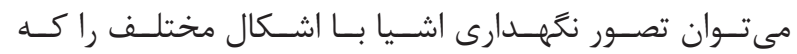

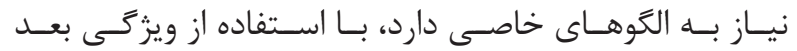

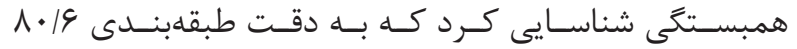

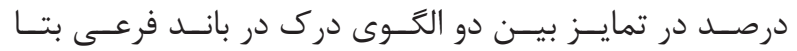

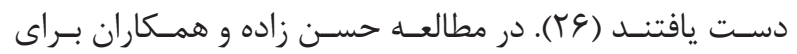

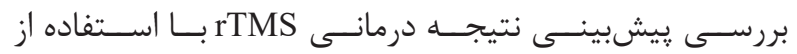

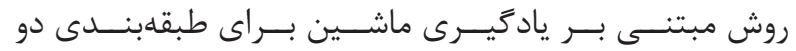

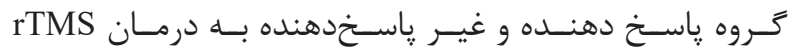

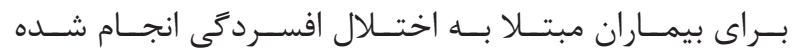

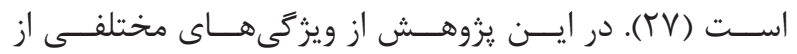

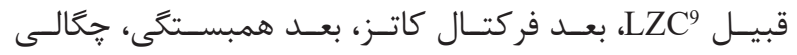

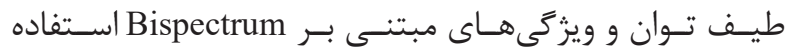

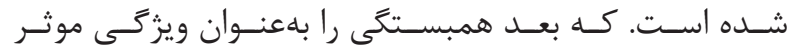

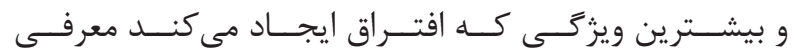

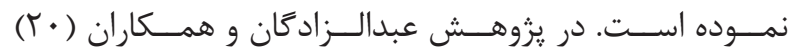

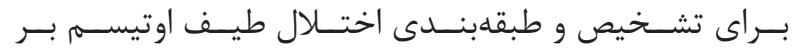

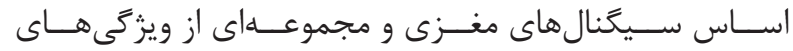

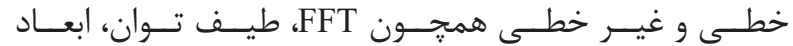

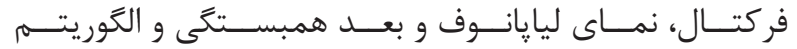

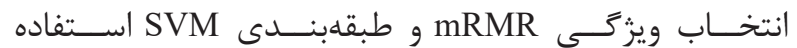

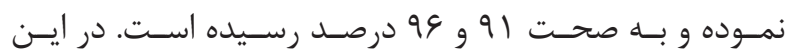

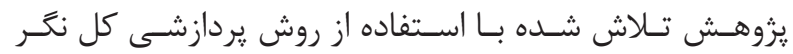

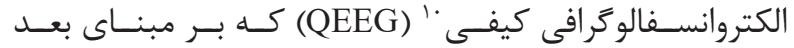

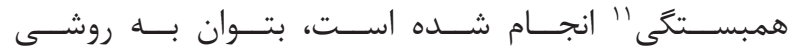

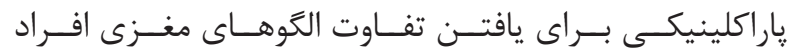

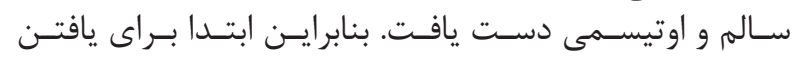

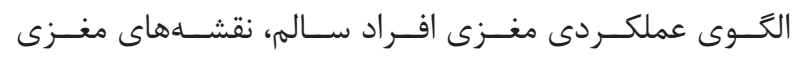

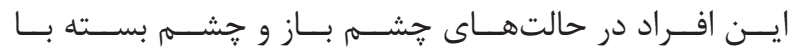

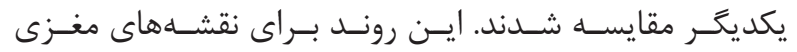

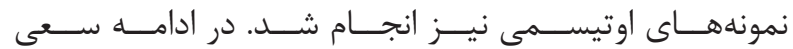

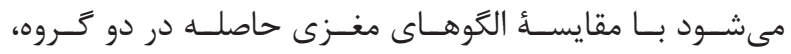

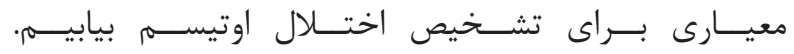

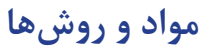

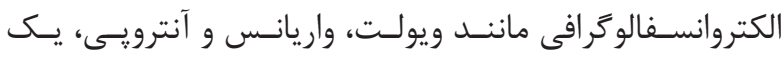

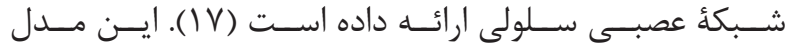

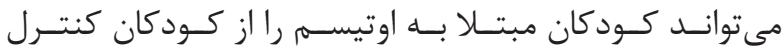

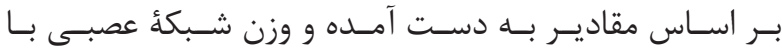

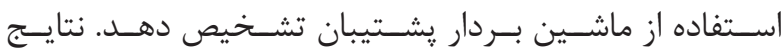

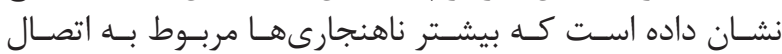

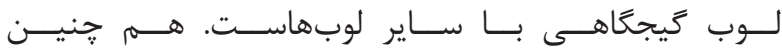

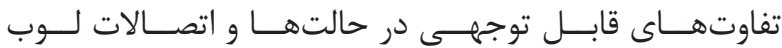

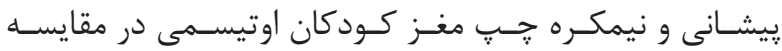

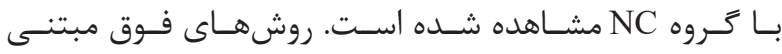

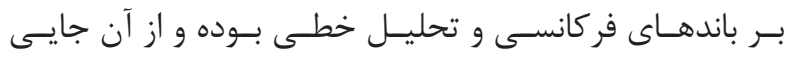

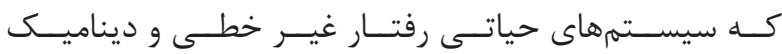

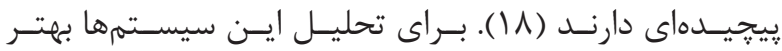

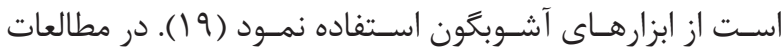

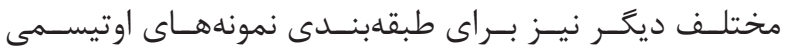

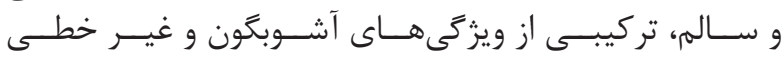

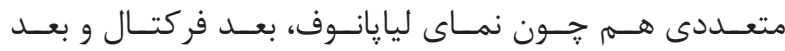

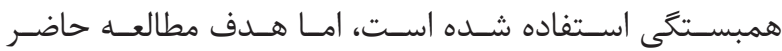

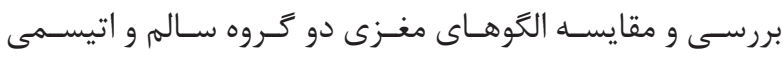

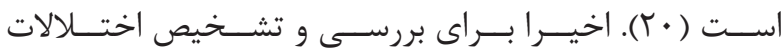

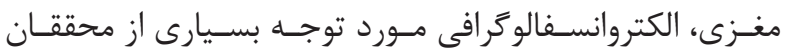

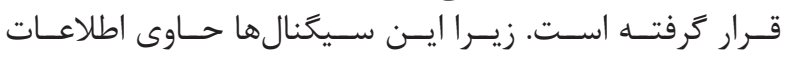

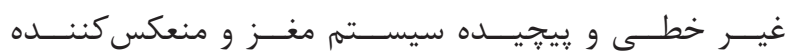

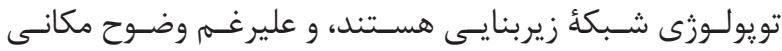

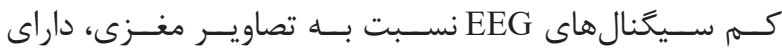

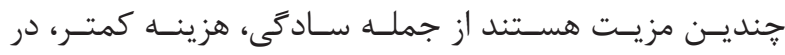

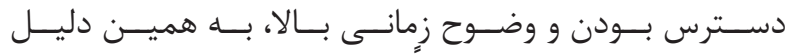

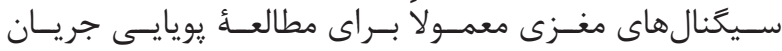

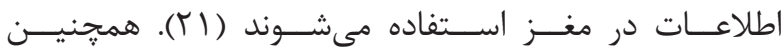

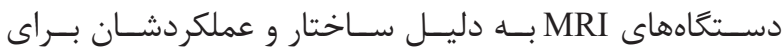

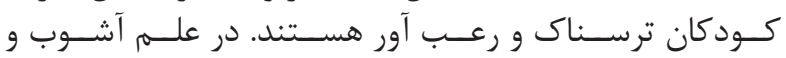

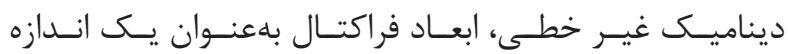

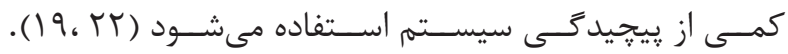

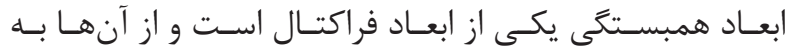

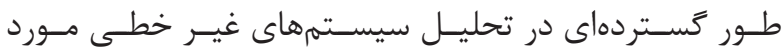

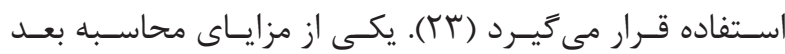

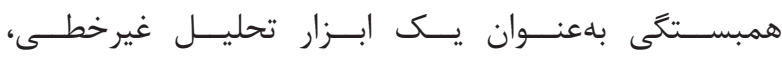

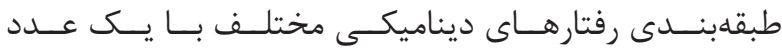

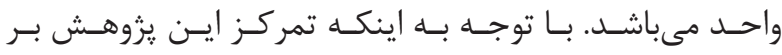

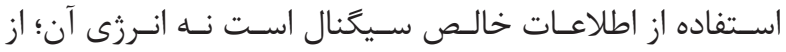

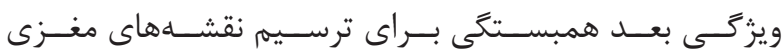

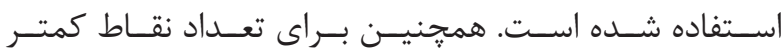

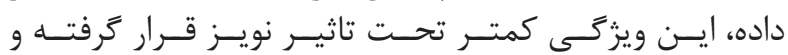

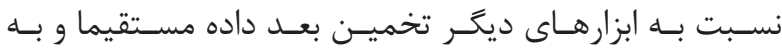

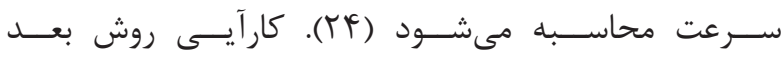

${ }^{9}$ Lempel-Ziv complexity

${ }^{10}$ Qualitative Electroencephalography

${ }^{11}$ Correlation Dimension 


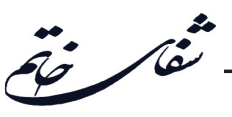

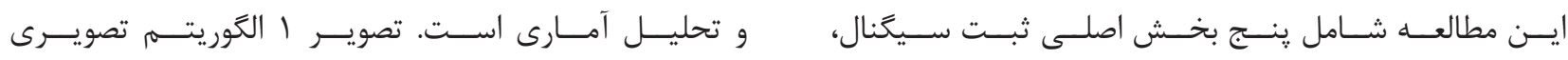

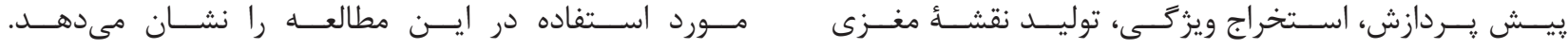

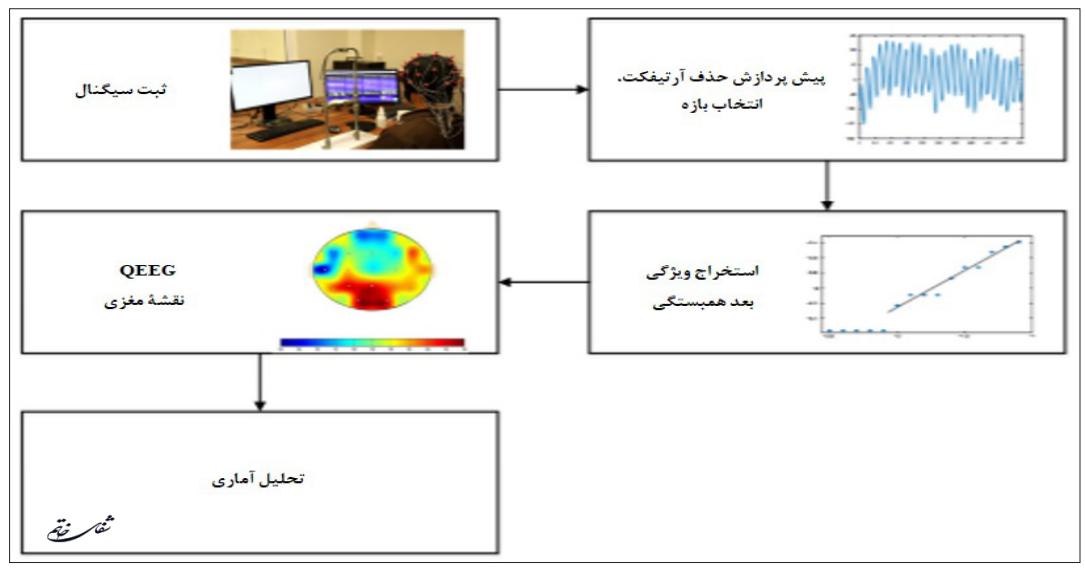

تصوير ا- الكَوريتم تصويرى از مراحل انجام اين ثروهش

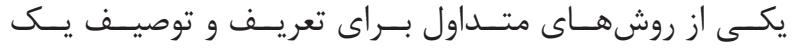

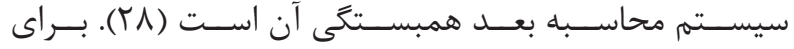

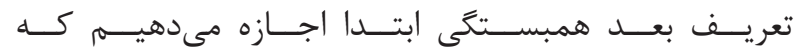

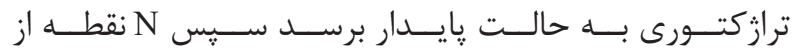

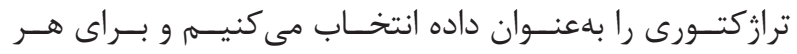

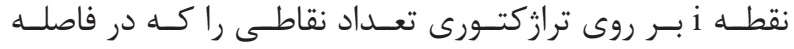

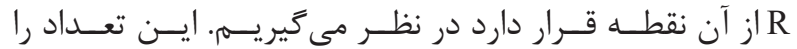

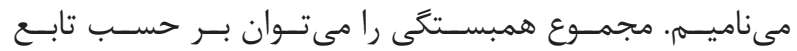

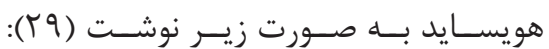

$\mathrm{C}(\mathrm{R})=\frac{1}{N(N-1)} \sum_{i=1}^{N} \sum_{\substack{J=1 \\ J \neq I}}^{N} \theta(R-|x i-x j|)(1, \Upsilon)$

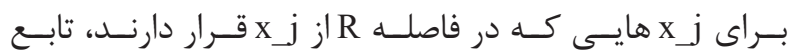

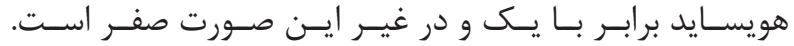

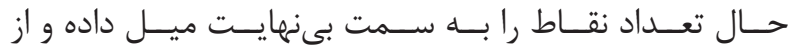

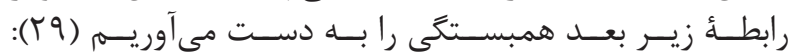

$$
\mathrm{Dc}=\lim _{R \rightarrow \mathrm{0}} \frac{\log C(R)}{\log R}(\mathrm{~T}, \mathrm{r})
$$

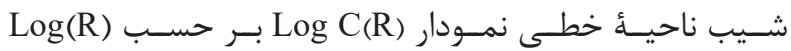

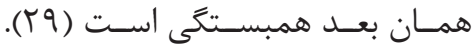

\section{نقشهُ مغزى}

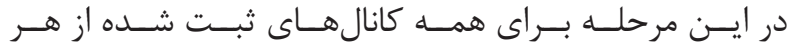

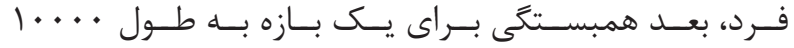

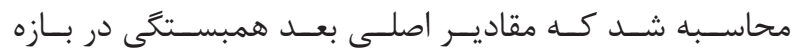

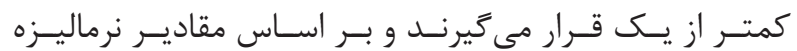

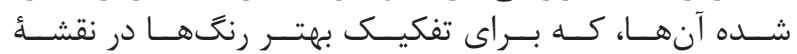

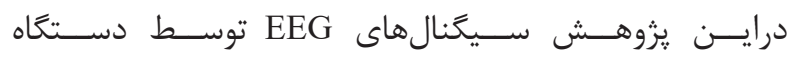
ewave32

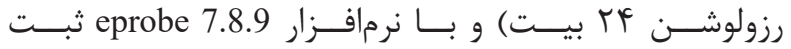

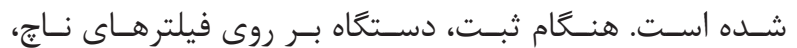

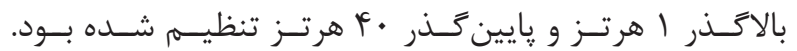

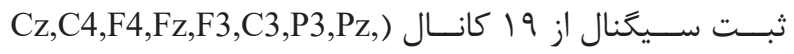

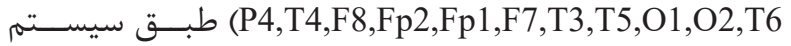

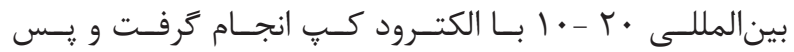

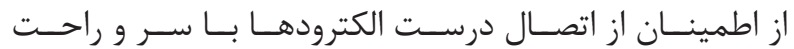

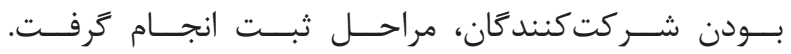

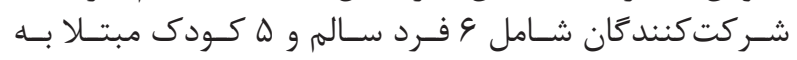

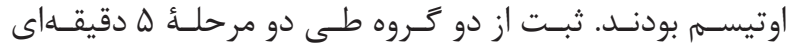

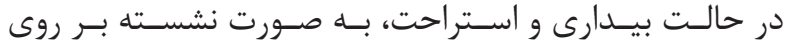

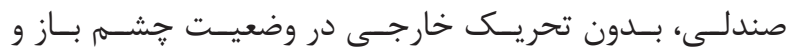

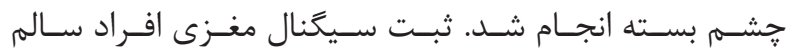

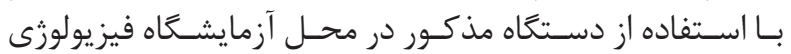

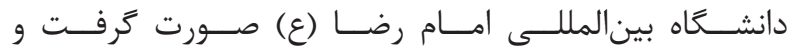

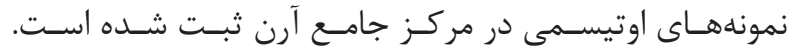

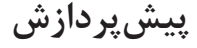

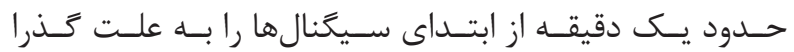

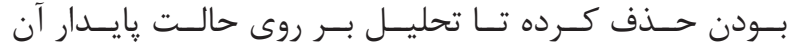

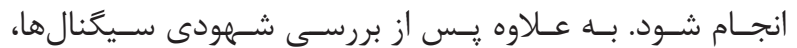

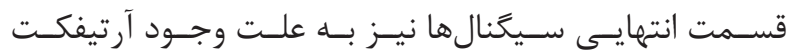

حـذف شــند. 


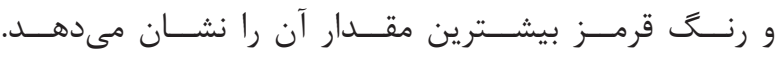

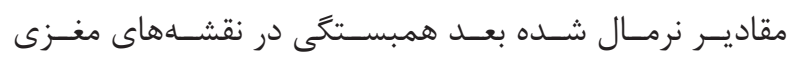

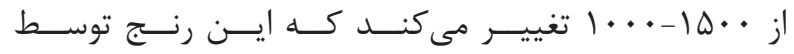

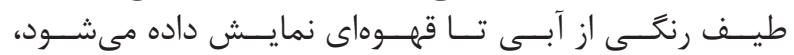

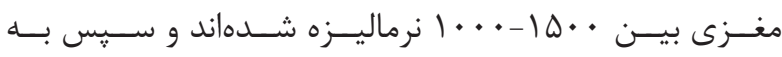

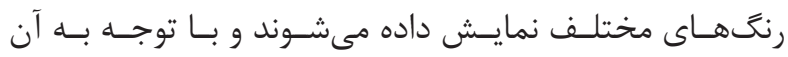

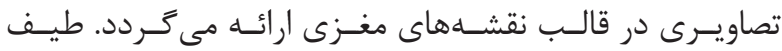

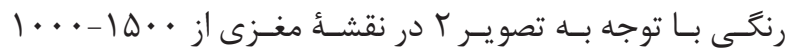

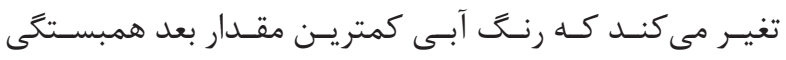

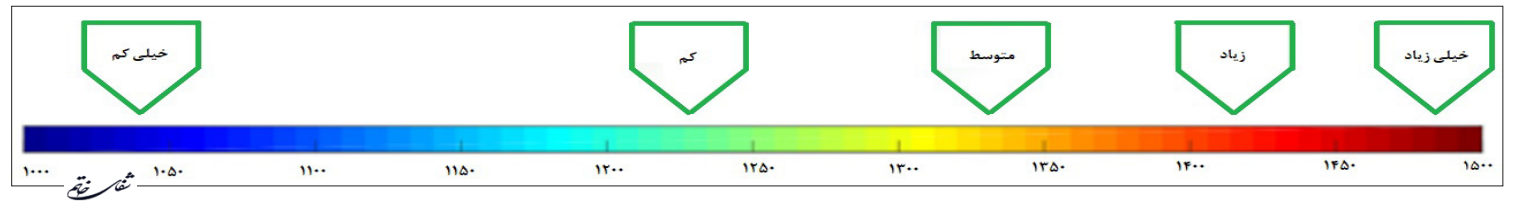

تصوير r- طيف رنكى نقشأ مغزى

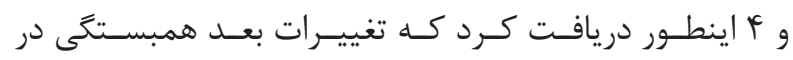

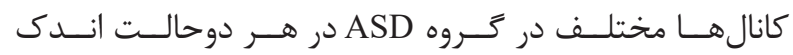

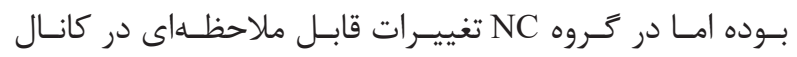

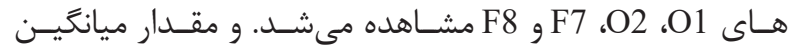

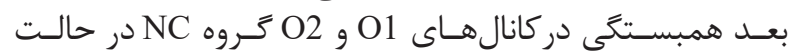

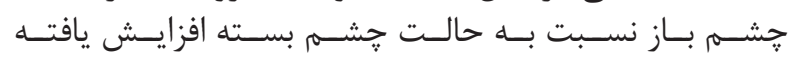

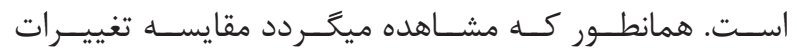

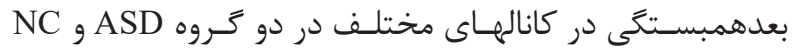

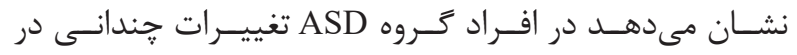

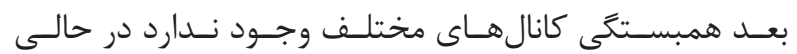

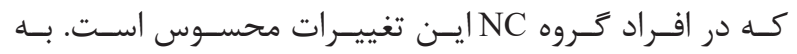

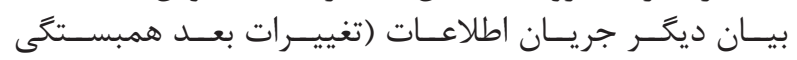

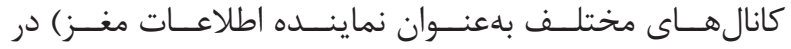

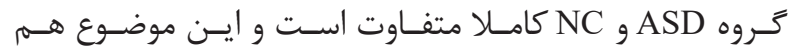

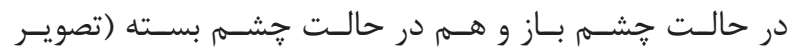

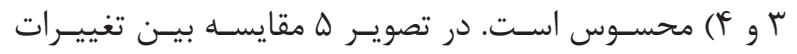

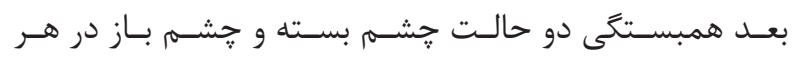

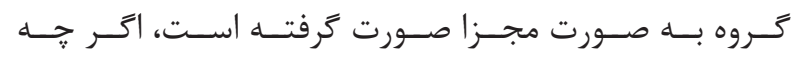

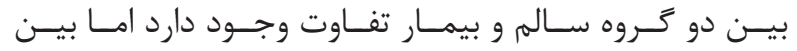

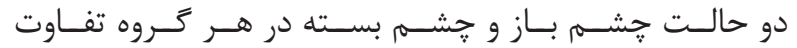

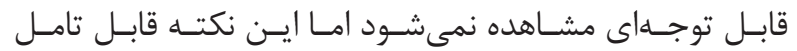

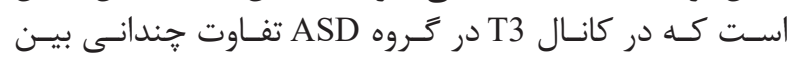

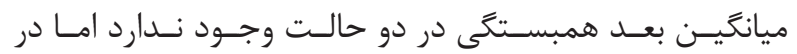

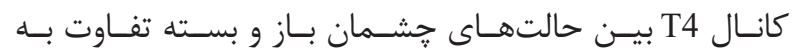

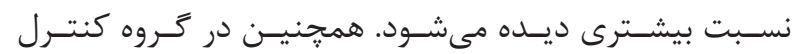

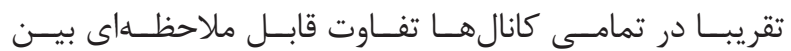

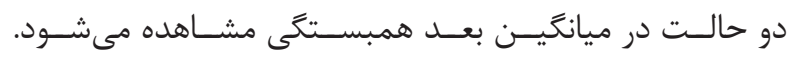

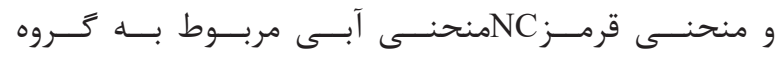

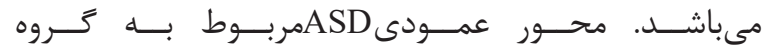

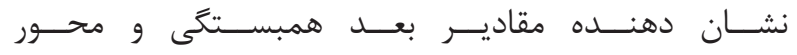

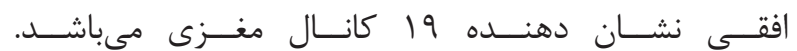

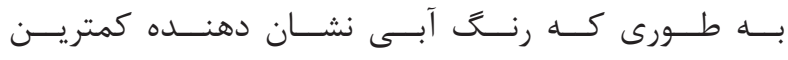

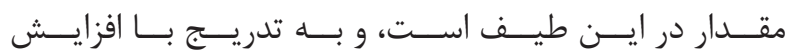

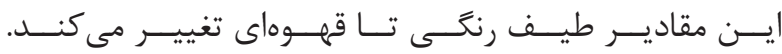

\section{تحليل آمارى}

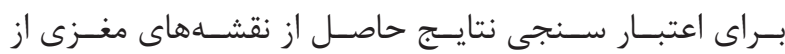

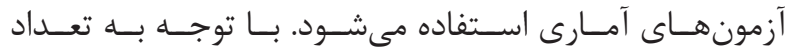

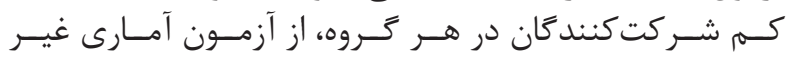

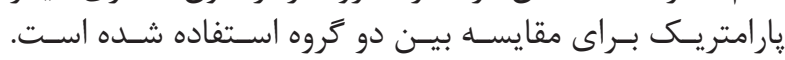

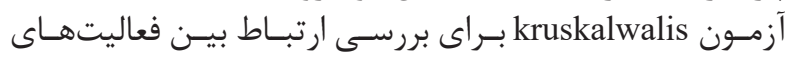

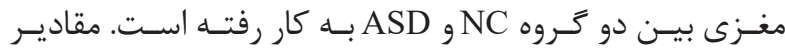

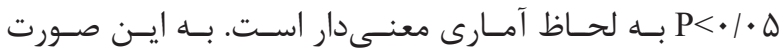

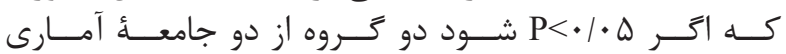

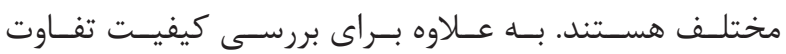

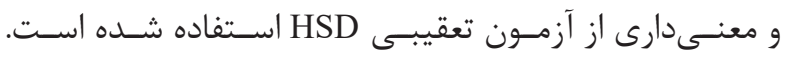

\section{يافتهها}

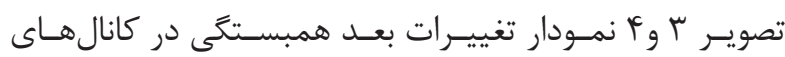

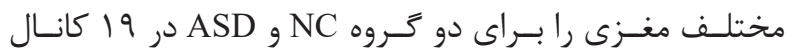

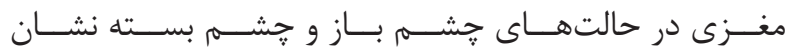

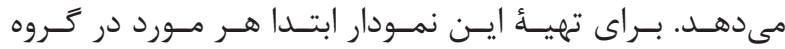
شـ

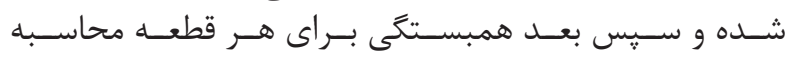

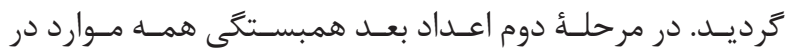

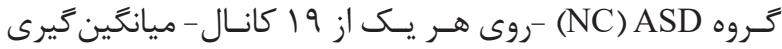

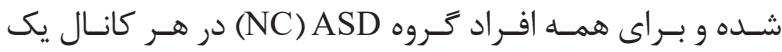

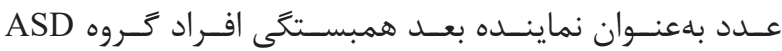

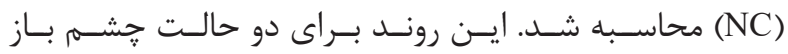

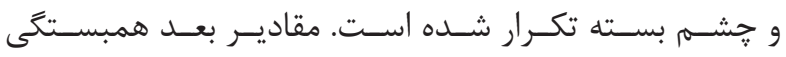

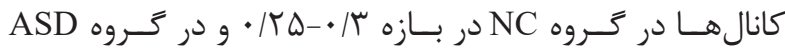

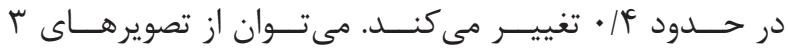




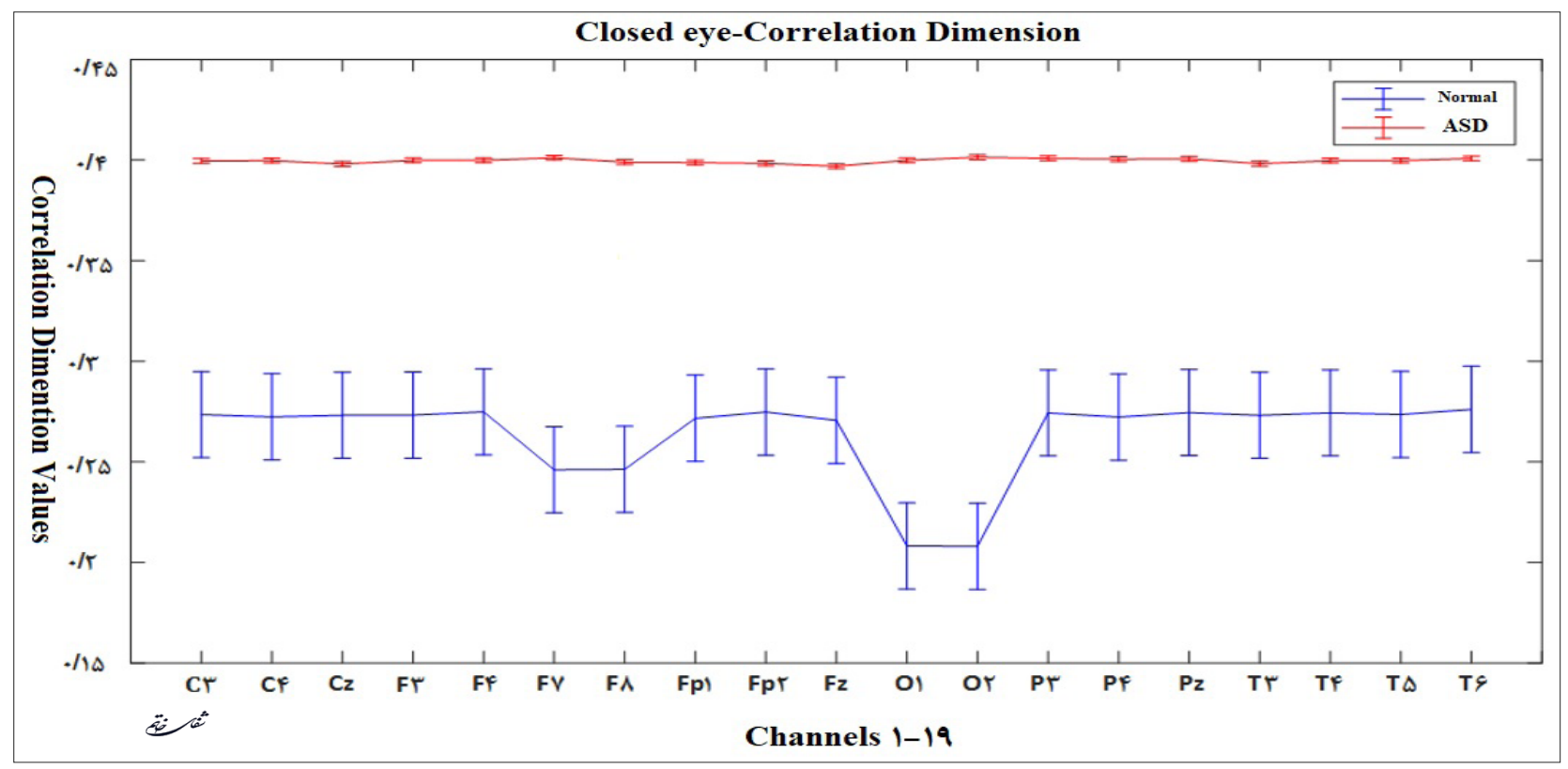

تصوير ץ- نمودار تغييرات بعد همبستىى در كانالهاى مختلف مغزى در حالت حشم بسته

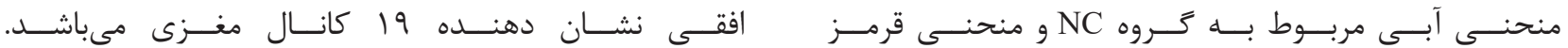

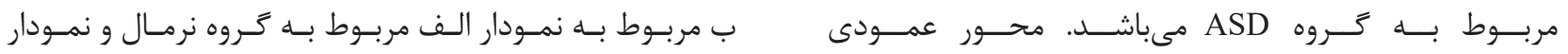

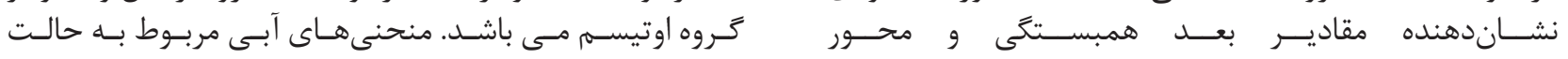

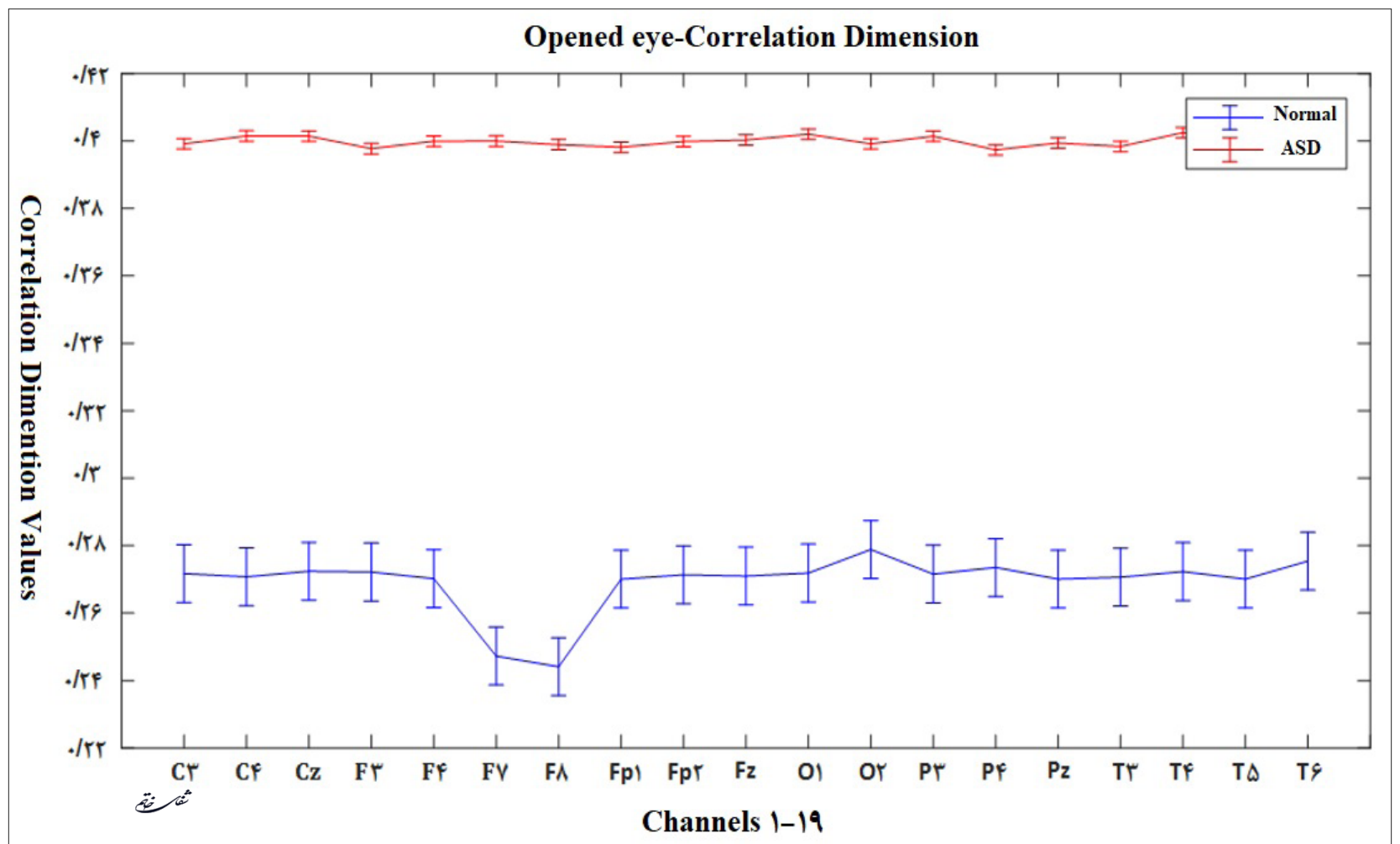



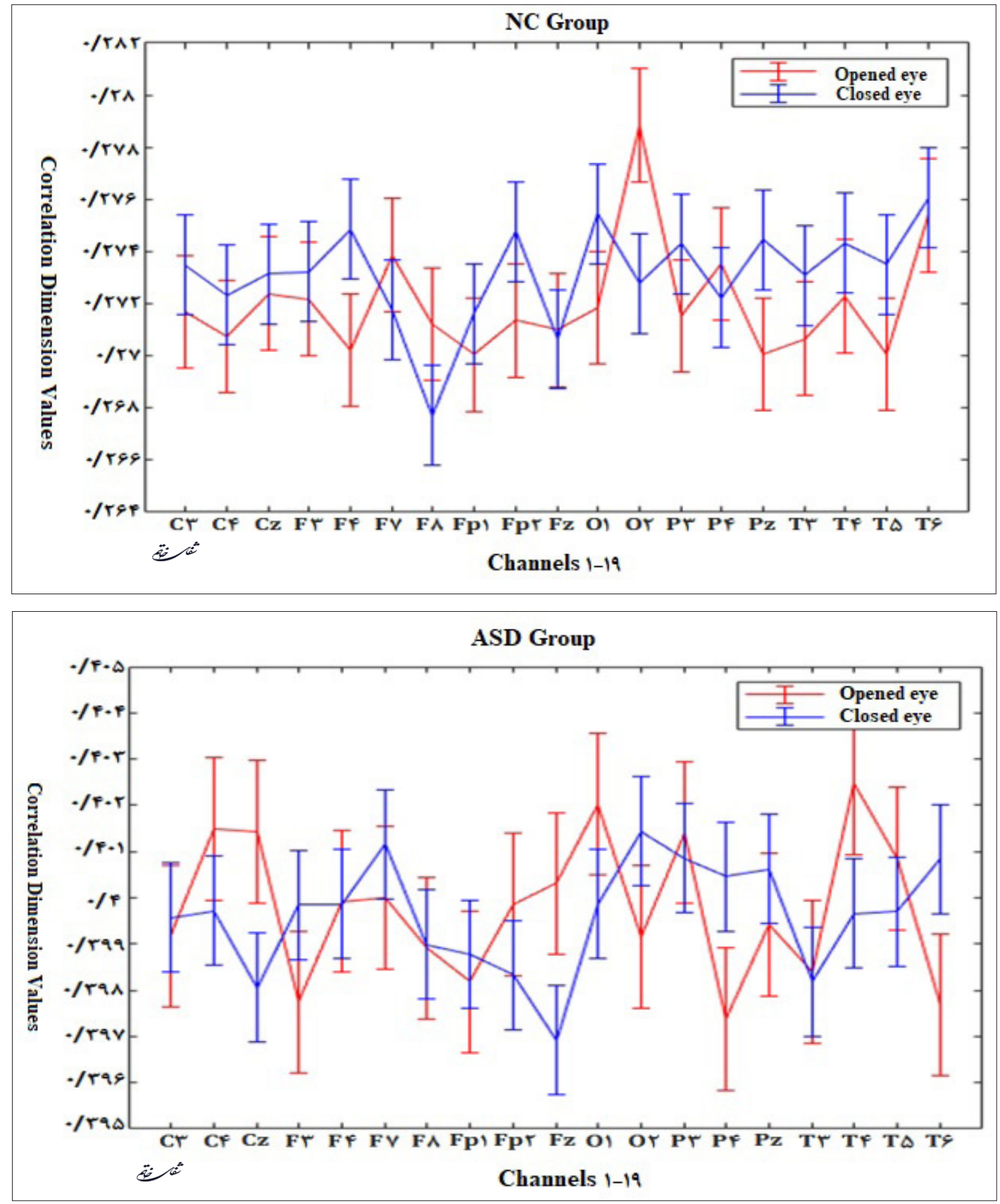

تصوير ه- نمودار تغييرات بعد همبستى در كانالهاى مختلف مغزى در حالت حشم بسته و حشم باز

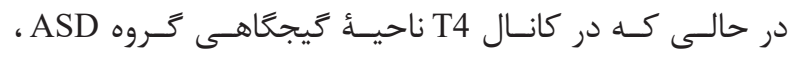

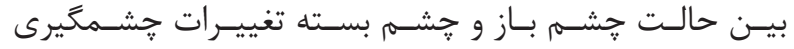

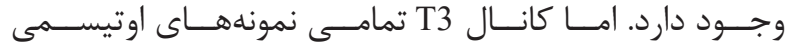

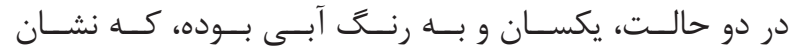

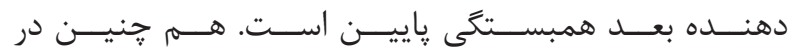

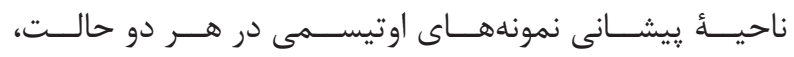

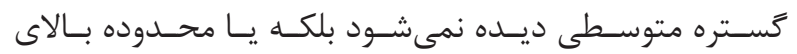

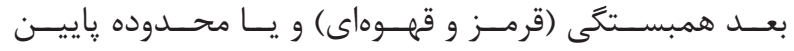

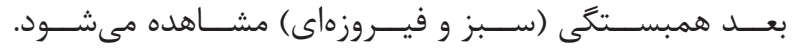

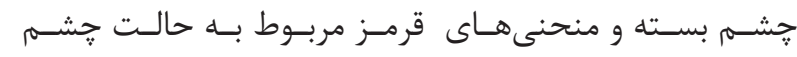

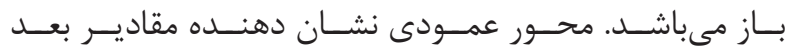

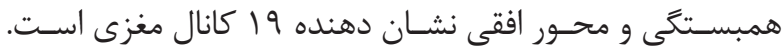
تحليل نقشأه مغزى

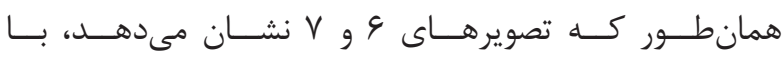

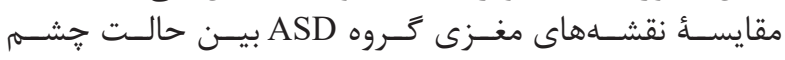

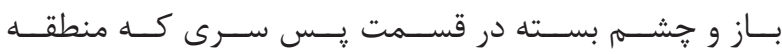

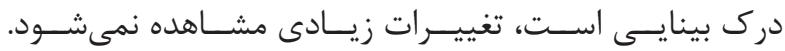



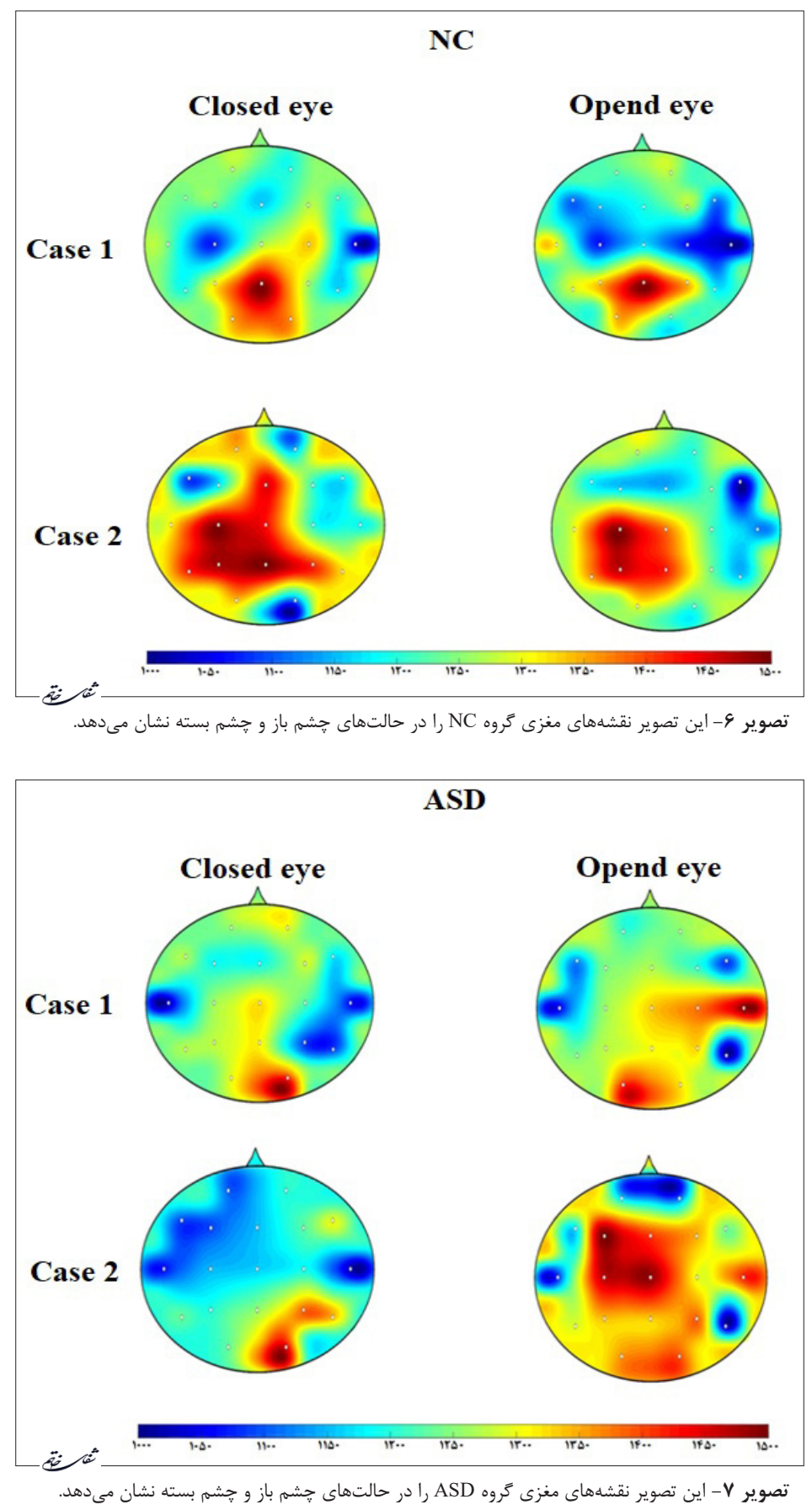

جشـم بــاز و خشــم بسـته از آزمــون kruskalwalis اسـتفاده

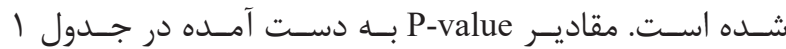

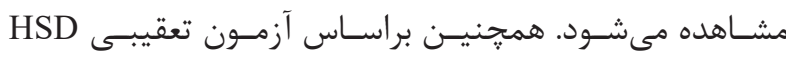

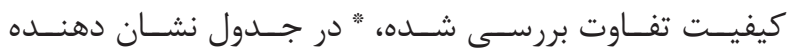

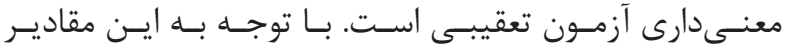

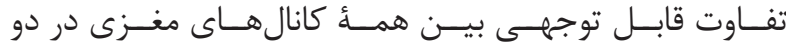

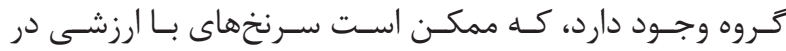

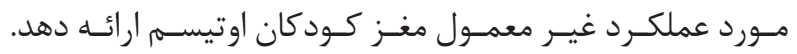

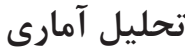

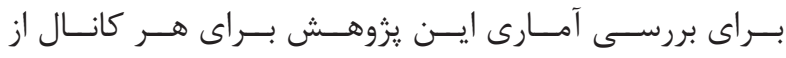

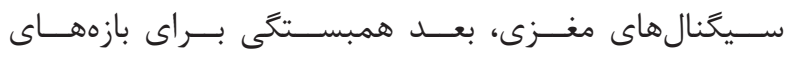

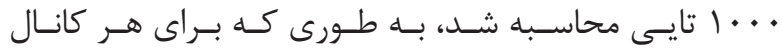

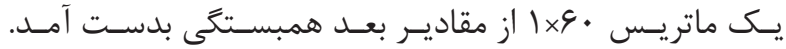

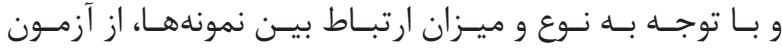

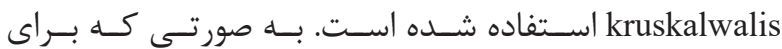

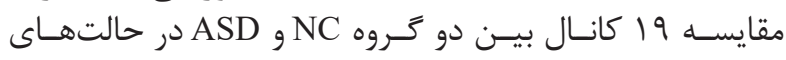


جدول ا- مقادير P-value كانال هاى مغزى در دو حالت جشم باز و حشم بسته در آزمون kruskalwalis

\begin{tabular}{|c|c|c|c|}
\hline \multicolumn{2}{|c|}{ Brain Channel } & P-Value in Closed eye State & P-Value in Opened eye State \\
\hline 1 & $\mathrm{Cr}$ & $3 \mid 1 / f q r V x^{10-1}$ & $31 / 99 V+x^{10-1}+$ \\
\hline r & $\mathrm{CF}$ & \$ $r / \Delta \varphi g \Delta x^{r l-1}$. & $* r / 19+r x^{\mid 9-1}$. \\
\hline$r$ & $\mathrm{Cz}$ & 3* $r(\Lambda \Lambda Y) x^{(f-1}$. & ; $\left.F / T r \wedge \Lambda x^{T \cdot-}\right)$. \\
\hline$f$ & Fr & $\omega \Delta / \cdot|q| x^{\mid \gamma-1}$. & $\% r / f \Delta \xi \wedge x^{1 T-} \mid$. \\
\hline$\Delta$ & Ff & * $r / \varepsilon \wedge \Delta r x^{\prime 1-}$. & $3 \mid r\left(\Delta r F+x^{\mid V-}\right)$. \\
\hline \& & FV & $\omega \Delta / \Delta \varphi+\Delta x^{r \mid-1}$. & $f \mid r / \cdot 1+r x^{\mid r-1}$ \\
\hline v & FA & * $N / 1+V \& x^{1 /-1}$. & * T/gY $\mid \Delta x^{\mid 9-1}$. \\
\hline$\Lambda$ & Fp) & * $r / V Y+9 x^{\mid Y-1}$. & H/ $r / 1 \Delta 99 x^{1 /-1}$ \\
\hline 9 & Fpr & |⿰亻丨 $Y(\Delta Q Y) x^{\mid r-1}$. & $* 1 / 9 r \wedge \Delta x^{\mid \gamma-1}$. \\
\hline $1 \cdot$ & $\mathrm{Fz}$ & $3 \mid k / F+V x^{\mid r-1}$ & $3\left|1 /+f \Delta \wedge x^{\mid 1-1}\right|+$ \\
\hline 11 & O) & $* 91.191 x^{14-1}$. & * $q\left(\Lambda F \mid Y x^{\mid A-1}\right.$. \\
\hline IT & Or & * r/rgr + $x^{\mid Y-1}$. & $* 1 / f r v g x^{1 T-1}$. \\
\hline Ir & Pr & W/rVIr $x^{\mid q-1}$. & 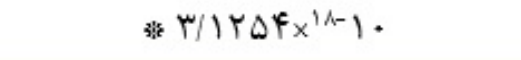 \\
\hline If & Pf & $\left.\neq 9(9) \cdot 9 x^{Y+-1}\right)$. & $31 / 94 r V x^{1 r-1}$. \\
\hline 10 & $\mathrm{Pz}$ & ; $(r / \Delta r)+x^{\mid r-1}$. & $* Y(\Delta) \wedge \xi x^{\mid A-1}$. \\
\hline 19 & $\mathrm{Tr}$ & $3 \mid / \Delta 9 \wedge+x^{\mid r-1}$. & $\% f / \wedge 90 \mid x^{19-1}$. \\
\hline iv & Tf & * $r|\wedge \xi| r x^{\mid r-1}$. & $* 4 / \ldots 9 V x^{19-1}$. \\
\hline 11 & $\mathrm{~T} \Delta$ & $3 ; r /+\Lambda r_{q} x^{\mid r-1}$. & $*|+| f \vee G x^{\mid A-1}$. \\
\hline 19 & T\% & क $1 / V+r g x^{\mid \gamma-1}$. & * $9 / 9 \vee Y^{\prime} q x^{\mid f-} \mid$. \\
\hline
\end{tabular}

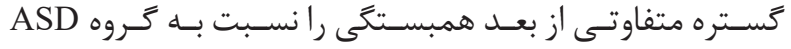

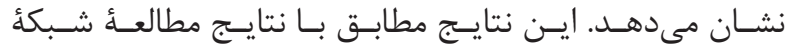

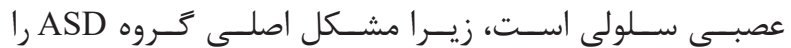

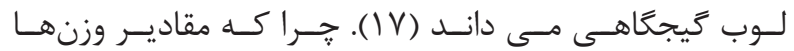

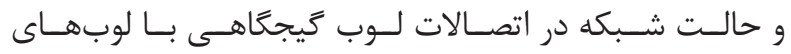

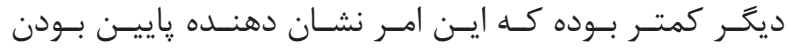

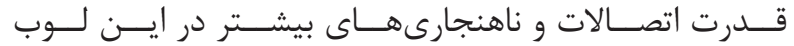

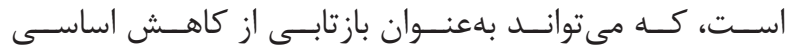

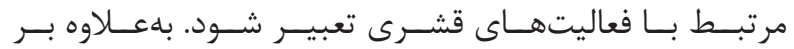

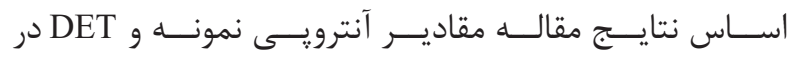

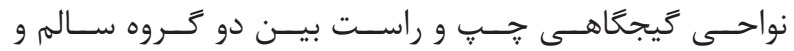

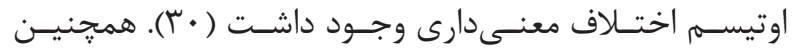

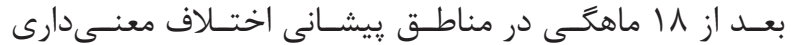

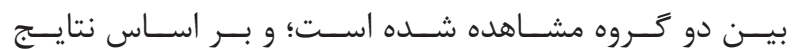

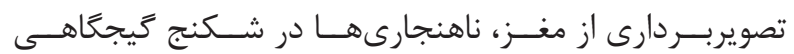

معنى دارى آزمون Post-hoc را مشخص مى كند كه بر اساس روش HSD انجام شده است.

بحث و نتيجه كيرى

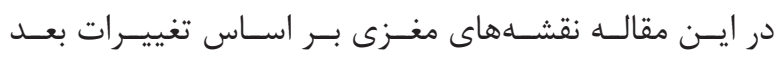

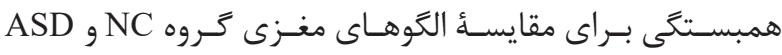

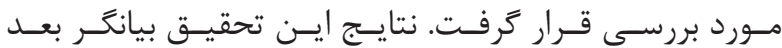

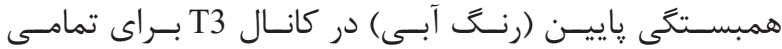

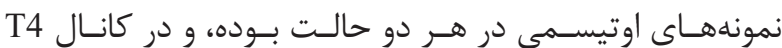

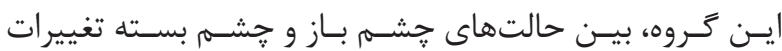

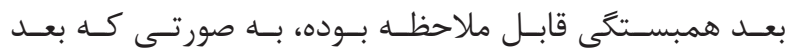

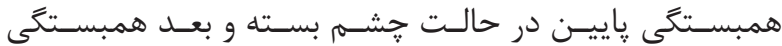

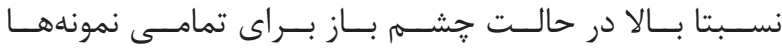

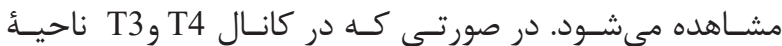

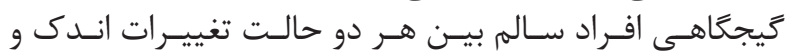




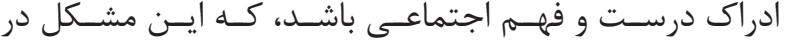

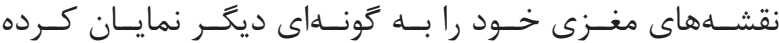

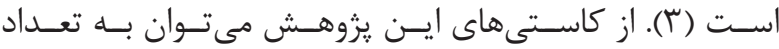

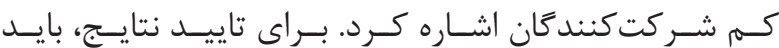

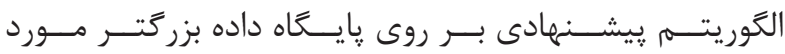

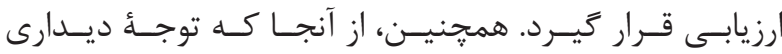

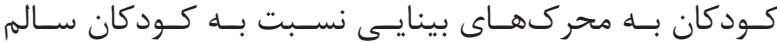

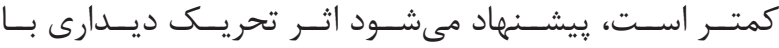

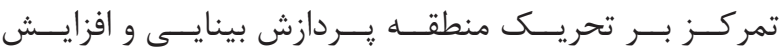

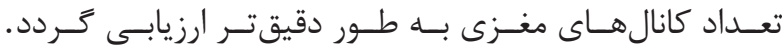

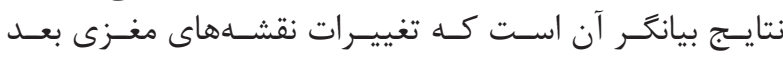

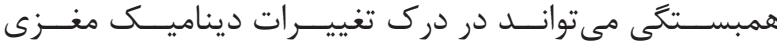

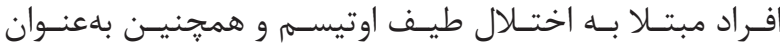

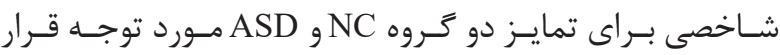

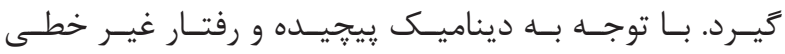

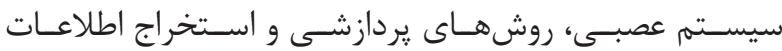

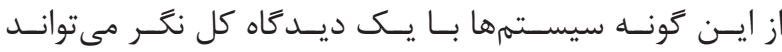

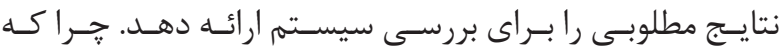

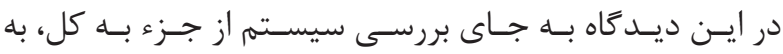

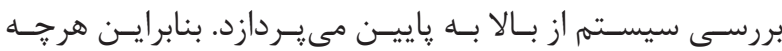

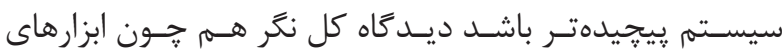

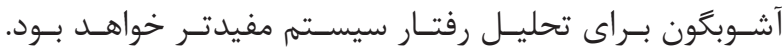

1. Sparks BF, Friedman SD, Shaw DW, Aylward EH, Echelard D, Artru AA, et al. Brain structural abnormalities in young children with autism spectrum disorder. Neurology. 2002; 59(2): 184-92.

2. Coben R. Connectivity-guided neurofeedback for autistic spectrum disorder. Biofeedback. 2007; 35(4): 131-5.

3. Wöhr M, Scattoni ML. Neurobiology of autism. Behavioural brain research. 2013; 251.

4. Landa RJ. Diagnosis of autism spectrum disorders in the first 3 years of life. Nature Clinical Practice Neurology. 2008; 4(3): 138-47.

5. Christensen DL, Braun KVN, Baio J, Bilder D, Charles J, Constantino JN, et al. Prevalence and characteristics of autism spectrum disorder among children aged 8 years-autism and developmental disabilities monitoring network, 11 sites, United States, 2012. MMWR Surveillance Summaries. 2018; 65(13):1.

6. Gray DE. Lay conceptions of autism: Parents' explanatory models. Medical Anthropology. 1994; 16(1-4): 99-118.

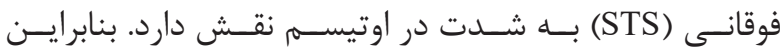

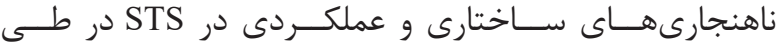

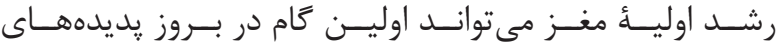

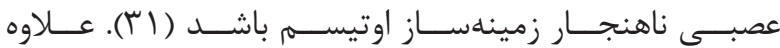

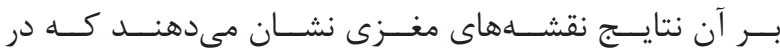

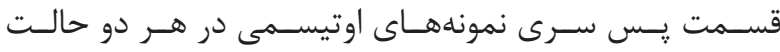

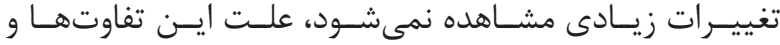

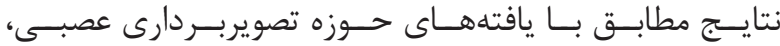

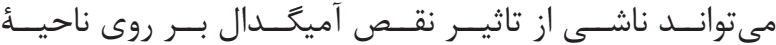

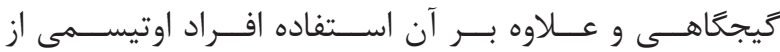

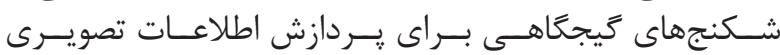

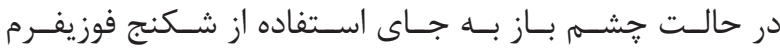

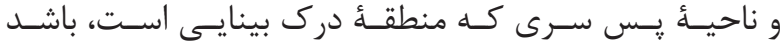

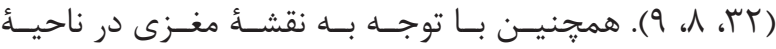

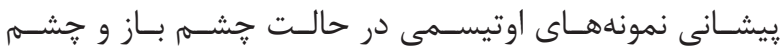

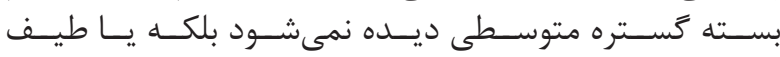

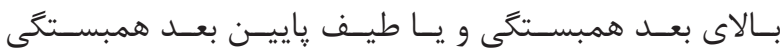

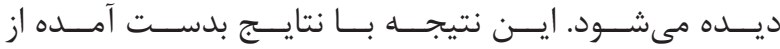

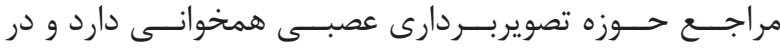

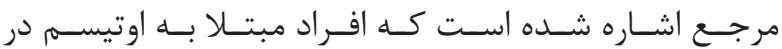

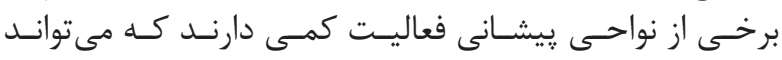

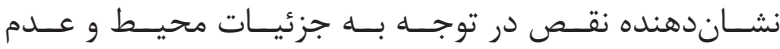

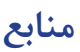

7. Hadjikhani N, Joseph RM, Snyder J, Chabris CF, Clark J, Steele S, et al. Activation of the fusiform gyrus when individuals with autism spectrum disorder view faces. Neuroimage. 2004; 22(3): 1141-50.

8. Park HR, Lee JM, Moon HE, Lee DS, Kim B-N, Kim J, et al. A short review on the current understanding of autism spectrum disorders. Experimental neurobiology. 2016; 25(1): 1-13.

9. Divanbeigi A, Divanbeigi A. A brief review on the causes of autism spectrum disorder. Shefaye Khatam. 2015; 3(1): 157-16.

10. Golinska AK. Detrended fluctuation analysis (DFA) in biomedical signal processing: selected examples. Stud Logic Grammar Rhetoric. 2012; 29: 107-15.

11. Liston C, Cohen MM, Teslovich T, Levenson D, Casey B. Atypical prefrontal connectivity in attention-deficit/ hyperactivity disorder: pathway to disease or pathological end point? Biological psychiatry. 2011; 69(12): 1168-77.

12. Schumann CM, Barnes CC, Lord C, Courchesne E. Amygdala enlargement in toddlers with autism related to severity of social and communication impairments. 
Biological psychiatry. 2009; 66(10): 942-9.

13. Pelphrey KA, Morris JP, McCarthy G. Neural basis of eye gaze processing deficits in autism. Brain. 2005; 128(5): 1038-48.

14. Dziobek I, Bahnemann M, Convit A, Heekeren HR. The role of the fusiform-amygdala system in the pathophysiology of autism. Archives of general psychiatry. 2010; 67(4): 397-405.

15. Elhabashy H, Raafat O, Afifi L, Raafat $\mathrm{H}$, Abdullah K. Quantitative EEG in autistic children. The Egyptian Journal of Neurology, Psychiatry and Neurosurgery. 2015; 52(3): 176.

16. Dimitrov PD, Petrov P, Aleksandrov I, Dimitrov I, Mihailova M, Radkova G, et al. Quantitative EEG Comparative Analysis between Autism Spectrum Disorder (ASD) and Attention Deficit Hyperactivity Disorder (ADHD). Journal of IMAB-Annual Proceeding Scientific Papers. 2017; 23(1): 1441-3.

17. Askari E, Setarehdan SK, Sheikhani A, Mohammadi MR, Teshnehlab M. Modeling the connections of brain regions in children with autism using cellular neural networks and electroencephalography analysis. Artificial intelligence in medicine. 2018; 89: 40-50.

18. Ma Y, Shi W, Peng C-K, Yang AC. Nonlinear dynamical analysis of sleep electroencephalography using fractal and entropy approaches. Sleep medicine reviews. 2018; 37: 85-93.

19. Rodriguez-Bermudez G, Garcia-Laencina PJ. Analysis of EEG signals using nonlinear dynamics and chaos: a review. Applied mathematics \& information sciences. 2015; 9(5): 2309.

20. Abdolzadegan D, Moattar MH, Ghoshuni M. A robust method for early diagnosis of autism spectrum disorder from EEG signals based on feature selection and DBSCAN method. Biocybernetics and Biomedical Engineering. 2020; 40(1): 482-93.

21. Bosl W, Tierney A, Tager-Flusberg H, Nelson C. EEG complexity as a biomarker for autism spectrum disorder risk. BMC medicine. 2011; 9(1): 18.

22. Sharma M, Pachori RB, Acharya UR. A new approach to characterize epileptic seizures using analytic time-frequency flexible wavelet transform and fractal dimension. Pattern Recognition Letters. 2017; 94: 172-9.

23. Natarajan K, Acharya R, Alias F, Tiboleng T, Puthusserypady SK. Nonlinear analysis of EEG signals at different mental states. Biomedical engineering online. 2004; 3(1): 7.

24. DeCoster GP, Mitchell DW. The efficacy of the correlation dimension technique in detecting determinism in small samples. Journal of Statistical Computation and Simulation. 1991; 39(4): 221-9.

25. Khalili Z, Moradi MH, editors. Emotion recognition system using brain and peripheral signals: using correlation dimensiontoimprovetheresultsofEEG.2009International Joint Conference on Neural Networks; 2009: IEEE.

26. Roy R, Sikdar D, Mahadevappa M. Chaotic behaviour of EEG responses with an identical grasp posture. Computers in Biology and Medicine. 2020: 103822.

27. Hasanzadeh F, Mohebbi M, Rostami R. Prediction of rTMS treatment response in major depressive disorder using machine learning techniques and nonlinear features of EEG signal. Journal of affective disorders. 2019; 256: 132-42.

28. Röschke J, Aldenhoff J. A nonlinear approach to brain function: deterministic chaos and sleep EEG. Sleep. 1992; 15(2): 95-101.

29. Hilborn RC. Chaos and nonlinear dynamics: an introduction for scientists and engineers: Oxford University Press on Demand; 2000.

30. Bosl WJ, Tager-Flusberg H, Nelson CA. EEG analytics for early detection of autism spectrum disorder: a datadriven approach. Scientific reports. 2018; 8(1): 1-20.

31. Zilbovicius M, Meresse I, Chabane N, Brunelle F, Samson Y, Boddaert N. Autism, the superior temporal sulcus and social perception. Trends in neurosciences. 2006; 29(7): 359-66.

32. Jambaque I, Mottron L, Ponsot G, Chiron C. Autism and visual agnosia in a child with right occipital lobectomy. Journal of Neurology, Neurosurgery \& Psychiatry. 1998; 65(4): 555-60. 